\title{
Determinants of long-term outcome in ICU survivors: results from the FROG-ICU study
}

Etienne Gayat ${ }^{1,28^{*}}$ (D) Alain Cariou ${ }^{2}$, Nicolas Deye ${ }^{3}$, Antoine Vieillard-Baron ${ }^{4}$, Samir Jaber $^{5}$, Charles Damoisel ${ }^{1}$, Qin Lu ${ }^{6}$, Xavier Monnet ${ }^{7}$, Isabelle Rennuit ${ }^{8}$, Elie Azoulay ${ }^{9}$, Marc Léone ${ }^{10}$, Heikel Oueslati $^{1}$, Bertrand Guidet ${ }^{11}$, Diane Friedman ${ }^{12}$, Antoine Tesnière $^{13}$, Romain Sonneville ${ }^{14}$, Philippe Montravers ${ }^{15}$, Sébastien Pili-Floury ${ }^{16}$, Jean-Yves Lefrant ${ }^{17,18}$, Jacques Duranteau ${ }^{19}$, Pierre-François Laterre ${ }^{20}$, Nicolas Brechot ${ }^{21}$, Karine Chevreul ${ }^{22}$, Morgane Michel ${ }^{23}$, Bernard Cholley ${ }^{24}$, Matthieu Legrand ${ }^{1}$, Jean-Marie Launay ${ }^{24}$, Eric Vicaut ${ }^{25}$, Mervyn Singer ${ }^{26}$, Matthieu Resche-Rigon ${ }^{27 \dagger}$ and Alexandre Mebazaa ${ }^{1+}$

\begin{abstract}
Background: Intensive care unit (ICU) survivors have reduced long-term survival compared to the general population. Identifying parameters at ICU discharge that are associated with poor long-term outcomes may prove useful in targeting an at-risk population. The main objective of the study was to identify clinical and biological determinants of death in the year following ICU discharge.
\end{abstract}

Methods: FROG-ICU was a prospective, observational, multicenter cohort study of ICU survivors followed 1 year after discharge, including 21 medical, surgical or mixed ICUs in France and Belgium. All consecutive patients admitted to intensive care with a requirement for invasive mechanical ventilation and/or vasoactive drug support for more than $24 \mathrm{~h}$ following ICU admission and discharged from ICU were included. The main outcome measure was all-cause mortality at 1 year after ICU discharge. Clinical and biological parameters on ICU discharge were measured, including the circulating cardiovascular biomarkers N-terminal pro-B type natriuretic peptide, high-sensitive troponin I, bioactive-adrenomedullin and soluble-ST2. Socioeconomic status was assessed using a validated deprivation index (FDep).

Results: Of 1570 patients discharged alive from the ICU, 333 (21\%) died over the following year. Multivariable analysis identified age, comorbidity, red blood cell transfusion, ICU length of stay and abnormalities in common clinical factors at the time of ICU discharge (low systolic blood pressure, temperature, total protein, platelet and white cell count) as independent factors associated with 1-year mortality. Elevated biomarkers of cardiac and vascular failure independently associated with 1-year death when they are added to multivariable model, with an almost 3-fold increase in the risk of death when combined (adjusted odds ratio 2.84 (95\% confidence interval 1.73-4.65), $p<0.001$ ).

Conclusions: The FROG-ICU study identified, at the time of ICU discharge, potentially actionable clinical and biological factors associated with poor long-term outcome after ICU discharge. Those factors may guide discharge planning and directed interventions.

Trial registration: ClinicalTrials.gov NCT01367093. Registered on 6 June 2011.

Keywords: Post-intensive care syndrome, Long-term survival, Biomarkers, Score, Discharge

\footnotetext{
* Correspondence: etienne.gayat@aphp.fr

'Equal contributors

'Department of Anesthesiology, Critical Care and Burn Unit, Hôpitaux

Universitaires Saint Louis-Lariboisière, Assistance Publique-Hôpitaux de

Paris, Université Paris Diderot—Paris 7, Sorbonne Paris Cité, UMR-S 942,

INSERM, Paris, France

${ }^{28}$ Department of Anesthesiology and Intensive Care, University Paris Diderot,

INSERM UMR-S 942, Saint Louis-Lariboisière University Hospitals, 2 rue

Ambroise Paré, 75010 Paris, France

Full list of author information is available at the end of the article
}

(c) The Author(s). 2018 Open Access This article is distributed under the terms of the Creative Commons Attribution 4.0 International License (http://creativecommons.org/licenses/by/4.0/), which permits unrestricted use, distribution, and reproduction in any medium, provided you give appropriate credit to the original author(s) and the source, provide a link to the Creative Commons license, and indicate if changes were made. The Creative Commons Public Domain Dedication waiver (http://creativecommons.org/publicdomain/zero/1.0/) applies to the data made available in this article, unless otherwise stated. 


\section{Background}

Survivors of critical illness will face a period of increased risk of reduced long-term survival and impaired quality of life compared to the general population [1]. This period, lasting several years, is associated with an increased risk of posttraumatic stress, depression, cognitive impairment and physical weakness, all grouped under the entity "postintensive care syndrome" (PICS) [2].

To reduce the mortality rate of intensive care unit (ICU) survivors, it is important to identify the group of patients who have a higher probability of death in the year following ICU discharge and to recognize the adjustable factors associated with mortality. Although data have been published regarding the long-term outcome of ICU patients, there are no recommendations for the long-term management of these patients. Only experts' opinions have been published [2, 3]. Some studies have demonstrated that mortality rates among ICU survivors are higher compared to the general population [4-8] and that an ICU stay impacts on patients' quality of life [9] and disability [10, 11]. Moreover, other studies [5, 6] found that this over-risk of mortality is sustained after 5-15 years of follow-up. Three studies $[4,7,8]$ reported a worse survival rate for ICU patients compared to an age-matched control population in the years following ICU discharge. Although we understand that age, comorbidity burden and severity of acute illness are important predictors of late mortality as described previously [12], we know less about clinical and laboratory values at the time of ICU discharge.

The transition of care from ICU to ward and, eventually, to home is a complex process with many challenges. We hypothesized that clinical and biological abnormalities present on the day of ICU discharge are associated with worse longterm outcome. In particular, we hypothesized that ICU survivors are at long risk of increased cardiovascular events, as suggested previously [13]. Among biological abnormalities, we focused on circulating cardiovascular biomarkers, namely N-terminal pro-B type natriuretic peptide (NTproBNP), high-sensitive troponin I (hs-TnI), bioactiveadrenomedullin (bio-ADM) and soluble-ST2 (sST2). The choice of those four biomarkers was guided by their relative function, with NT-proBNP a marker of cardiac congestion, hs-TnI a marker of cardiac injury, sST2 a marker of cardiac remodeling and bio-ADM a marker of vascular dysfunction.

Accordingly, the FROG-ICU (French and European Outcome reGistry in Intensive Care Units) study aimed to identify clinical and biological (including cardiovascular biomarkers) parameters associated with long-term outcome in ICU survivors.

\section{Methods}

\section{Study design}

FROG-ICU was a prospective, observational, multicenter cohort study in which survivors of critical illness were followed up for up to 1 year post ICU discharge. The study was conducted in France and Belgium in accordance with Good Clinical Practice (Declaration of Helsinki 2002) and Ethical Committee approvals (Comité de Protection des Personnes-Ile de France IV, IRB n00003835 and Commission d'éthique biomédicale hospitalo-facultaire de l'hôpital de Louvain, IRB $\mathrm{n}^{\circ}$ B403201213352). It is registered on ClinicalTrials.gov (NCT01367093). Patients were included from August 2011 to June 2013. Details of design and methods have been published previously [14]. All patients admitted to any of the participating centers during the recruitment period who met the eligibility criteria and survived their ICU stay had a clinical examination and biological tests performed at discharge from the ICU, and were followed up for 1 year through telephone calls and postal questionnaires at 3, 6 and 12 months.

\section{Participants}

The study involved 21 medical, surgical or mixed ICUs in 14 university hospitals. Inclusion criteria were: invasive mechanical ventilation support for at least $24 \mathrm{~h}$ and/or treatment with a vasoactive agent (except dopamine) for more than $24 \mathrm{~h}$. Noninclusion criteria were: age younger than 18 years old; severe head injury (initial Glasgow Coma Scale $\leq 8$ ), brain death or a persistent vegetative state; pregnancy or breastfeeding; transplantation in the past 12 months; moribund patient; and/or no social security coverage. The Ethical Committees waived the need for written consent; all patients and/or next of kin were informed and oral consent was documented in the patients' medical records by the investigator.

\section{Study objectives}

The primary purpose of the FROG-ICU study was to assess the incidence of all-cause mortality in the year following ICU discharge, and to identify independent factors associated with mortality. The main secondary objective of FROG-ICU was to evaluate the association between circulation cardiovascular biomarkers levels at discharge and 1-year mortality.

\section{Data collection}

Details of data collection have been reported previously [14]. Briefly, clinical and biological data were recorded at admission, during the ICU stay and at discharge from the ICU. In order to explore the mechanisms of clinical abnormalities at ICU discharge associated with subsequent deaths, cardiovascular biomarkers were collected at discharge and measured centrally. These included markers of: cardiac failure (NT-proBNP; Roche Diagnostics GmbH, Mannheim, Germany); cardiac ischemia (hsTnI; Abbott, Abbott Park, IL, USA); vascular 
dysfunction [15] (bio-ADM; Adrenomed GmbH, Hennigsdorf, Germany); and cardiac stress (sST2; Eurobio, Critical Diagnostics, San Diego, CA, USA) which prognosticates for cardiovascular death [16, 17]. The deprivation index (FDep) was used as a measure of socioeconomic inequalities in health status. The FDep is based on the patients' residential zip codes and was specifically developed for the French context using the following four variables to compute a single composite index: median household income, percentage of high school graduates in the population aged $\geq 15$ years, percentage of blue-collar workers in the active population and unemployment rate [18].

\section{Statistical analysis}

Results are expressed as median (interquartile range (IQR)) or count (percentage) as appropriate. The primary analysis examining factors associated with 1 -year mortality was based on analysis of the clinical and biological variables measured in patients discharged alive from the ICU. Marginal associations between single variables and 1-year mortality were assessed by a Wilcoxon rank-sum test for quantitative variables and the chi-square test for qualitative variables. Multivariable logistic regression was used to determine a set of variables independently associated with 1 -year mortality. Variables associated with outcome at a 0.05 level and with less than $20 \%$ of missing data were considered within the multivariable model. The loglinearity of the quantitative variables was evaluated systematically, and, if appropriate, variable transformation was performed. Log-linearity of the association between continuous variables and the outcome was checked using a cubic spline and the Wald test. Cutoff values were derived from the plots of the effect according to the value of the variable of interest. Missing values were handled by multiple imputation by chained equations (MICE) [19]. All variables selected for the multivariable model were considered in the imputation model. A total of 51 imputed samples was generated using 15 iterations of the chained equation process. A selection model process was performed using a backward stepwise approach with stopping rules based on a cutoff at 0.05 for $p$ values. At each step of the selection, inference was combined from the sets of imputed samples using Rubin's rules [20]. The existence of any colinearities was observed, and a test of goodness of fit was performed using the Hosmer-Lemeshow test on the complete case model [21]. Measures of association consisted of odds ratios (ORs) and their confidence intervals (CIs) at 95\% estimated using Rubin's rules. The predictive power of the four biomarkers of interest was assessed using receiver operating curve (ROC) analyses. The area under the ROC (AUC) was estimated for each biomarker. For both the clinical model and the clinical model including biomarker information, the AUCs were estimated from the sets of imputed samples using Rubin's rules. The latter two were compared using the Delong test. As it is now recognized that highlighting a statistically significant association between new biomarkers and patient outcomes is not sufficient to demonstrate the interest of these biomarkers in terms of risk prediction [22-24], we used the proposed methodology of Pencina et al. [23], which has been used in multiple articles of application. The net reclassification improvement (NRI) and integrated discrimination improvement (IDI) of each biomarker added to the full clinical model will be calculated, and comparisons between different biomarkers will be performed [23].

Calculating the number of subjects required was based on the primary endpoint; that is, the risk factors associated with 1-year all-cause mortality. Study of the literature and preliminary studies conducted in December 2009 in 14 participating centers led us to estimate a 1year mortality after ICU discharge of $18 \%$. To ensure detection with a power of $80 \%$ for the detection of binary prognostic factors with a prevalence of $33 \%$ and an expected OR of 1.5 in a population with a probability of death in the year following ICU discharge of approximately 18\%, 1636 patients were required [25]. Assuming a $10 \%$ rate of refusal and/or loss to follow-up, the number of patients to be enrolled was raised to 1800 . Finally, since the expected in-ICU mortality rate was $25 \%$, the total number of patients included in the study was 2250 . $p<0.05$ was considered statistically significant. All statistical analyses were performed using $\mathrm{R}$ statistical software version 3.1.1 or above (The "R" Foundation for Statistical Computing, Vienna, Austria).

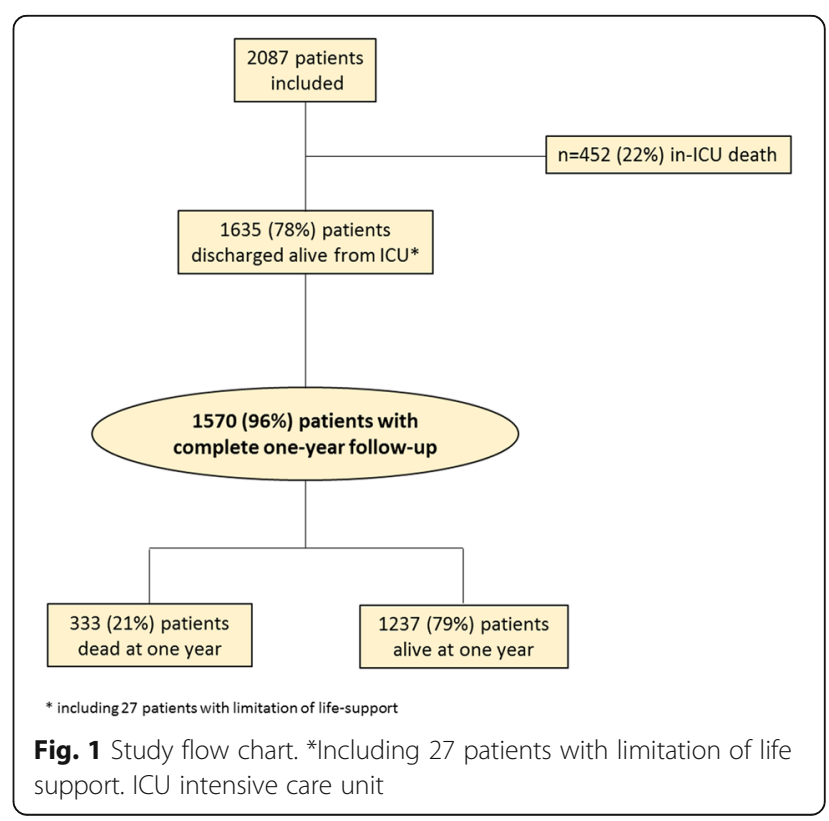


Table 1 Patient characteristics

\begin{tabular}{|c|c|c|c|c|c|}
\hline \multirow[t]{2}{*}{ Variable } & \multirow{2}{*}{$\begin{array}{l}\% \text { of } \\
\text { missing } \\
\text { value }\end{array}$} & \multirow{2}{*}{$\begin{array}{l}\text { Studied patients } \\
(n=1570)\end{array}$} & \multicolumn{2}{|l|}{1 year post ICU } & \multirow[t]{2}{*}{$p$ value } \\
\hline & & & Survivors $(n=1237)$ & Nonsurvivors $(n=333)$ & \\
\hline Age (years) & 0.0 & $61(49 ; 73)$ & $58(47 ; 70)$ & $71(61 ; 79)$ & $<0.01$ \\
\hline Male gender & 0.0 & $1000(63.7)$ & $782(63 \%)$ & $218(66 \%)$ & 0.48 \\
\hline BMI $\left(\mathrm{kg} / \mathrm{m}^{2}\right)$ & 37.5 & $26(23 ; 31)$ & $26(23 ; 31)$ & $26(23 ; 31)$ & 0.96 \\
\hline Charlson score & 0.1 & $3(1 ; 4)$ & $2(1 ; 4)$ & $4(3 ; 6)$ & $<0.01$ \\
\hline Deprivation index (FDep) & 10.8 & $-0.6(-1.6 ; 0.3)$ & $-0.6(-1.6 ; 0.3)$ & $-0.6(-1.5 ; 0.4)$ & 0.44 \\
\hline SOFA score at admission & 37.2 & $6(4 ; 9)$ & $7(4 ; 10)$ & $8(5 ; 11)$ & 0.11 \\
\hline SAPS I| score at admission & 0.1 & $46(34 ; 59)$ & $45(33 ; 58)$ & $51(40 ; 65)$ & $<0.01$ \\
\hline Main cause of admission & & & & & $<0.01$ \\
\hline Septic shock & 0.1 & $339(22 \%)$ & $244(20 \%)$ & 95 (29\%) & \\
\hline Acute respiratory failure & & 303 (19\%) & $230(19 \%)$ & $73(22 \%)$ & \\
\hline Acute neurological disorder & & $241(15 \%)$ & $210(17 \%)$ & $31(9 \%)$ & \\
\hline Out-of-hospital cardiac arrest & & $117(8 \%)$ & $102(8 \%)$ & $15(5 \%)$ & \\
\hline \multicolumn{6}{|l|}{ In-ICU management } \\
\hline In-ICU LOS (days) & 0.0 & $12(7 ; 21)$ & $12(7 ; 20)$ & $13(7 ; 24)$ & 0.03 \\
\hline In-hospital LOS (days) & 0.1 & $26(15 ; 43)$ & $25(15 ; 43)$ & $28(16 ; 47)$ & 0.05 \\
\hline Tracheotomy & 0.0 & $241(15 \%)$ & 181 (15\%) & $60(18 \%)$ & 0.13 \\
\hline RRT & 0.0 & $286(18 \%)$ & $202(16 \%)$ & 84 (25\%) & $<0.01$ \\
\hline Inotrope/vasopressor & 0.0 & 1151 (73\%) & $888(72 \%)$ & $263(79 \%)$ & $<0.01$ \\
\hline $\mathrm{RBC}$ & 0.0 & $676(43 \%)$ & $490(40 \%)$ & $186(56 \%)$ & $<0.01$ \\
\hline FFP & 0.0 & $236(15 \%)$ & $171(14 \%)$ & $65(20 \%)$ & $<0.01$ \\
\hline \multicolumn{6}{|l|}{ Status at discharge } \\
\hline $\mathrm{SBP}(\mathrm{mmHg})$ & 12.9 & $125(111 ; 139)$ & $125(112 ; 139)$ & $122(108 ; 139)$ & 0.03 \\
\hline $\mathrm{DBP}(\mathrm{mmHg})$ & 16.7 & $68(59 ; 76)$ & $69(60 ; 77)$ & $64(55 ; 73)$ & $<0.01$ \\
\hline $\mathrm{HR}(\mathrm{bpm})$ & 14.1 & $90(79 ; 101)$ & $90(79 ; 100)$ & $89(79 ; 101)$ & 0.41 \\
\hline Atrial fibrillation & 10.0 & 297 (21\%) & $265(21 \%)$ & $32(21.2)$ & 0.96 \\
\hline Temperature $\left({ }^{\circ} \mathrm{C}\right)$ & 10.3 & $37.1(36.8 ; 37.5)$ & $37.1(36.8 ; 37.5)$ & $37(36.6 ; 37.4)$ & $<0.01$ \\
\hline Sodium (mmol/l) & 3.1 & $139(136 ; 142)$ & $139(136 ; 142)$ & $139(136 ; 142)$ & 0.6 \\
\hline Potassium (mmol/l) & 8.3 & $3.9(3.6 ; 4.2)$ & $3.9(3.6 ; 4.2)$ & $4.0(3.6 ; 4.2)$ & 0.36 \\
\hline Creatinine $(\mu \mathrm{mol} / \mathrm{l})$ & 3.7 & $66(51 ; 95)$ & $64(50 ; 87)$ & $80(57 ; 131)$ & $<0.01$ \\
\hline $\mathrm{eGFR}\left(\mathrm{ml} / \mathrm{min} / 1.73 \mathrm{~m}^{2}\right)$ & 3.7 & $91(51.2 ; 110)$ & $110(75 ; 146)$ & $79(46 ; 119)$ & $<0.01$ \\
\hline Lactate (mmol/l) & 58.3 & $1.0(0.7 ; 1.3)$ & $1.0(0.7 ; 1.3)$ & $1.1(0.8 ; 1.4)$ & $<0.01$ \\
\hline WBC count $\left(/ \mathrm{mm}^{3}\right)$ & 13.6 & $9600(7015 ; 13,100)$ & $9500(7000 ; 12,952)$ & $10050(7342 ; 13,962)$ & 0.04 \\
\hline Hemoglobin (g/dl) & 13.6 & $10.0(9.0 ; 11.2)$ & $10.2(9.1 ; 11.3)$ & $9.6(8.7 ; 10.6)$ & $<0.01$ \\
\hline Platelets count $\left(/ \mathrm{mm}^{3}\right)$ & 12.9 & $291,500(181,750 ; 432,500)$ & $308,500(191,000 ; 457,000)$ & $240500(137,500 ; 347,750)$ & $<0.01$ \\
\hline Bilirubin $(\mathrm{mmol} / \mathrm{l})$ & 63.2 & $11(7 ; 20)$ & $10(7 ; 19)$ & $14(9 ; 36)$ & $<0.01$ \\
\hline Glycemia (mmol/l) & 16.8 & $6.8(5.7 ; 8.3)$ & $6.7(5.7 ; 8.2)$ & $7.1(5.9 ; 8.7)$ & 0.01 \\
\hline Total protein (g/L) & 18.6 & $62(56 ; 69)$ & $63(57 ; 69)$ & $60(52 ; 66)$ & $<0.01$ \\
\hline
\end{tabular}

Results expressed as count (percentage) or median (interquartile range)

BMI body mass index, SOFA Sequential Organ Failure Assessment, SAPS Simplified Acute Physiology Score, ICU intensive care unit, LOS length of stay, RRT renal replacement therapy, $R B C$ red blood cell transfusion, FFP fresh frozen plasma transfusion, $S B P$ systolic blood pressure, DBP diastolic blood pressure, $H R$ heart rate, eGFR estimated glomerular filtration rate, WBC white blood cell

\section{Results}

Of the 2087 ICU patients who consented to participate in the FROG-ICU study, 1570 were discharged from the
ICU and followed up for 1 year (Fig. 1). Patient characteristics are presented in Table 1. ICU mortality was $22 \%$. Median ICU and hospital lengths of stay for ICU 
survivors were 12 (IQR 7; 21) and 26 (IQR 15; 43) days, respectively. Details of patients' comorbidities are presented in Additional file 1: Table S1. The main reasons for ICU admission were septic shock (22\%), acute respiratory failure (19\%), acute neurological disorder (15\%) and out-of-hospital cardiac arrest (8\%). On admission, the Sequential Organ Failure Assessment (SOFA) score was 6 (IQR 4; 9) and the Simplified Acute Physiologic Score (SAPS) II was 46 (IQR 34; 59).

Clinical and biological characteristics at the time of ICU discharge were generally in the normal range (Table 1), except for hemoglobin (median value $10 \mathrm{~g} / \mathrm{dl}$ ). Patients were mostly discharged to a ward $(n=976$, $50 \%)$ or step-down unit $(n=269,14 \%)$.

\section{Determinants of 1-year survival after ICU discharge}

Of the 1570 ICU survivors, 333 (21\%) died during the year following ICU discharge, including 123 (8\%) during the index hospitalization (Additional file 1: Figure S1). Univariate analysis revealed that the 333 nonsurvivors at 1 year post ICU discharge had a greater degree of illness severity at ICU admission and more comorbidities (Table 1, Additional file 1: Table S1). One-year nonsurvivors were more likely to have septic shock as the cause of admission. While in the ICU, 1-year nonsurvivors required more renal replacement therapy, inotropes/vasopressors and transfusion than survivors. On ICU discharge, nonsurvivors had lower blood pressure and residual organ dysfunction than survivors. Yet renal function was more profoundly altered in nonsurvivors with a higher serum creatinine and lower eGFR at ICU discharge (Table 1).
Multivariable analysis identified 14 independent predictors of post-ICU survival (Fig. 2). Odds ratios of significantly associated variables are presented in Additional file 1: Table S2. Linearity of the association between continuous variables in the multivariable model and the outcome is depicted in Additional file 1: Figure S2. The area of the ROC curve for the multivariable model was 0.787 (95\% CI 0.759-0.815). Age and comorbidities (Charlson comorbidity score, vascular disease, severe valvular disease, chronic kidney diseases, cancer and loss of autonomy) were associated with a greater 1year risk of death. At ICU discharge, five clinical variables (low values of systolic blood pressure, body temperature, total protein and platelet counts, and a high white blood cell count) were associated with an increased post-ICU risk of death. With respect to their ICU stay, red blood cell transfusion and prolonged ICU length of stay were associated with higher risk of 1-year post-ICU mortality. Of note, AUCs of SOFA at admission and SAPS II were 0.574 (95\% CI 0.531-0.619) and 0.605 (95\% CI $0.572-0.64$ ) respectively; both were significantly lower than the AUC of the clinical score.

\section{Association between cardiovascular biomarkers at discharge and 1-year survival after ICU discharge}

At the time of ICU discharge, 1-year nonsurvivors had elevated levels of all measured cardiovascular biomarkers (Table 2). As depicted in Additional file 1: Figure S3, the association between the level of biomarkers at discharge and the outcome was not linear in all cases. After dichotomization according to the median value, elevated biomarkers of cardiac (NT-proBNP, sST2) and vascular

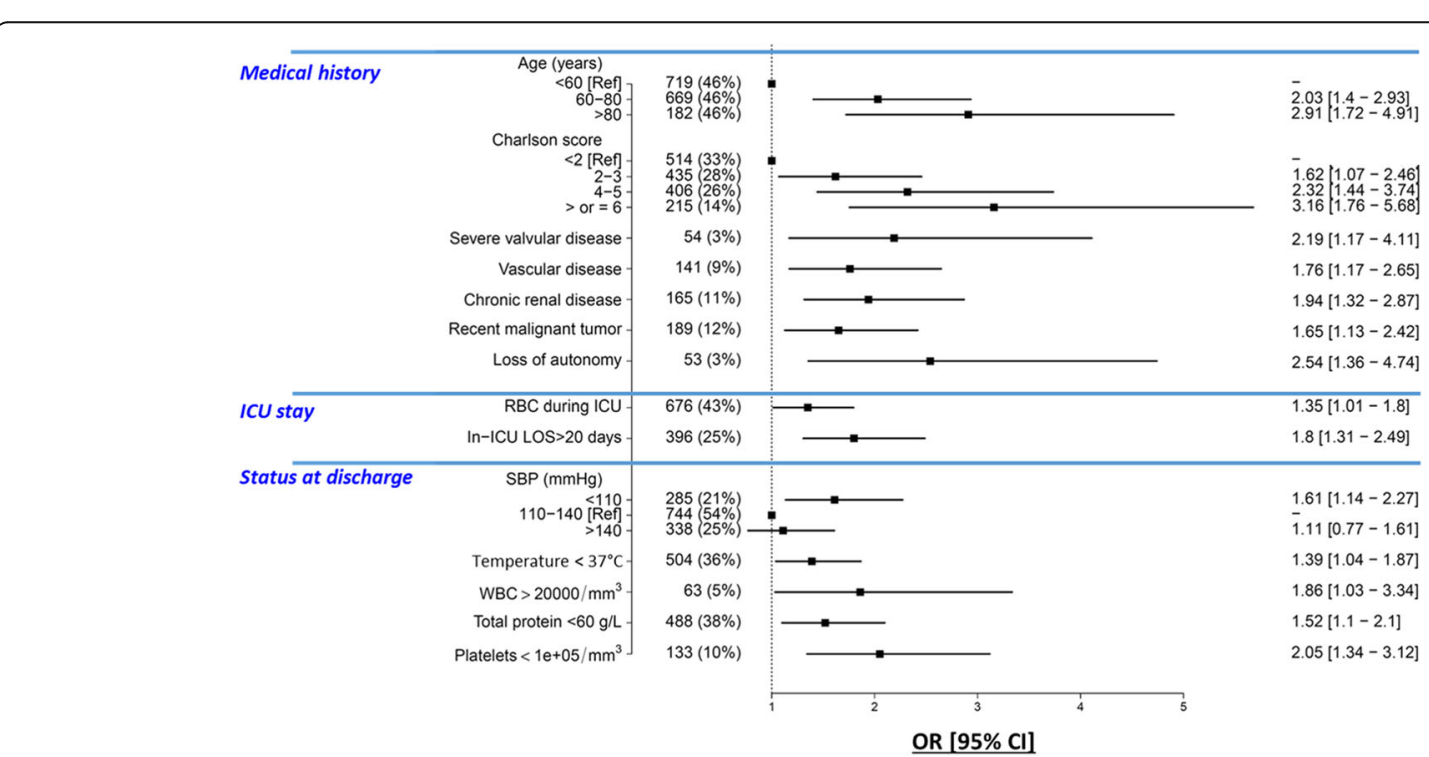

Fig. 2 Clinical predictors of 1-year post-ICU survival. Area under the ROC curve of the multivariable model including the 14 variables is 0.787 (95\% CI 0.759-0.815). RBC red blood cell transfusion, ICU intensive care unit, LOS length of stay, SBP systolic blood pressure, WBC white blood cell, Ref reference, $\mathrm{OR}$ odds ratio, $\mathrm{Cl}$ confidence interval 
Table 2 Performance of cardiovascular biomarkers measured at ICU discharge for the prediction of 1-year post-ICU survival

\begin{tabular}{|c|c|c|c|c|}
\hline & NT-proBNP (pg/ml) & hs-Tnl (pg/ml) & bio-ADM (pg/ml) & sST2 (ng/ml) \\
\hline Normal value & $<300$ & $<14$ & $<43$ & $<23.6$ for $\mathrm{men} /<16.0$ for women \\
\hline \multicolumn{5}{|l|}{ Missing data (\%) } \\
\hline \multicolumn{5}{|l|}{ Median (IQR) at discharge } \\
\hline All patients $(n=1570)$ & $541(149 ; 2073)$ & $11.2(4.2 ; 39.2)$ & $33.3(20.2 ; 60.5)$ & $122.2(73.1 ; 208.8)$ \\
\hline 1-year survivor $(n=1237)$ & $464(127 ; 1681)$ & $9.7(3.7 ; 33.8)$ & $30.5(18.7 ; 52.3)$ & $112.5(67.9 ; 188.6)$ \\
\hline 1-year nonsurvivor $(n=333)$ & $1471(371 ; 5719)$ & $18.5(7.6 ; 74.7)$ & $50.4(28.5 ; 107.9)$ & $189.1(102.4 ; 301.2)$ \\
\hline \multicolumn{5}{|c|}{ Association with prognosis (OR (95\% Cl) of biomarker > median) } \\
\hline Univariate analysis & $2.50(1.85-3.38)$ & $2.03(1.51-2.73)$ & $2.52(1.86-3.42)$ & $2.44(1.81-3.30)$ \\
\hline Multivariable analysis & $2.05(1.33-3.18)$ & $1.41(0.94-2.13)$ & $1.61(1.06-2.45)$ & $1.53(1.01-2.33)$ \\
\hline \multicolumn{5}{|l|}{ AUC of ROC curve $(95 \% \mathrm{Cl})$} \\
\hline Biomarker alone & $0.659(0.619-0.699)^{*}$ & $0.625(0.588-0.663)^{*}$ & $0.672(0.635-0.711)^{*}$ & $0.657(0.618-0.697)^{*}$ \\
\hline Biomarker + clinical score & $0.794(0.766-0.823)^{* *}$ & $0.789(0.759-0.817)$ & $0.794(0.766-0.822)$ & $0.800(0.773-0.827)^{* *}$ \\
\hline \multicolumn{5}{|c|}{ NRI of biomarkers added to the full clinical model } \\
\hline$\%$ Events to higher risk & 44.3 & 37.4 & 43.4 & 42.5 \\
\hline$\%$ Nonevents to higher risk & 14.8 & 16.1 & 15.7 & 17.2 \\
\hline$\%$ Events to lower risk & 55.7 & 62.6 & 56.6 & 57.5 \\
\hline$\%$ Nonevents to lower risk & 85.2 & 83.9 & 84.3 & 82.8 \\
\hline Total NRI for events $(95 \% \mathrm{Cl})$ & $-0.115(-0.263$ to 0.033$)$ & $-0.253(-0.397$ to -0.109$)$ & $-0.133(-0.281$ to 0.015$)$ & $-0.149(-0.296$ to -0.003$)$ \\
\hline $\begin{array}{l}\text { Total NRI for nonevents } \\
(95 \% \mathrm{Cl})\end{array}$ & $0.705(0.650-0.759)$ & $0.678(0.622-0.734)$ & $0.686(0.631-0.742)$ & $0.656(0.598-0.714)$ \\
\hline Total cNRI (95\% CI) & $0.590(0.433-0.747)$ & $0.425(0.271-0.580)$ & $0.554(0.396-0.711)$ & $0.507(0.349-0.664)$ \\
\hline \multicolumn{5}{|c|}{ IDI of biomarkers added to the full clinical model } \\
\hline Events to higher risk & 0.014 & -0.009 & 0.018 & 0.005 \\
\hline Nonevents to lower risk & 0.023 & 0.016 & 0.024 & 0.02 \\
\hline Total (95\% Cl) & $0.036(0.019-0.054)$ & $0.007(0-0.014)$ & $0.041(0.023-0.06)$ & $0.024(0.009-0.04)$ \\
\hline
\end{tabular}

NT-proBNP N-terminal pro-B type natriuretic peptide, $h s$-Tnl hyper-sensitive troponin I, bio-ADM bio-adrenomedullin, $s S T 2$ soluble ST2, IQR interquartile range, AUC area under the curve, $O R$ odds ratio, $R O C$ receiver operating curve, $C l$ confidence interval, $N R I$ net reclassification improvement, $I D I$ integrative discrimination improvement

${ }^{*} p<0.05$ corresponding to Wilcoxon test comparing survivors to nonsurvivors

${ }^{* *} p<0.05$ corresponding to DeLong test comparing $c$-statistics of multivariate clinical model without (see Fig. 2) and with inclusion of the biomarker

(bio-ADM) failure were independently associated with 1-year death when they are added to the multivariable model, with an almost 3-fold increase in the risk of death when combined (adjusted OR 2.84 (95\% CI 1.734.65), $p<0.001$ ) (Fig. 3). Of note, the association between elevated hs-TnI and 1-year mortality did not remain significant after adjustment. Although only NTproBNP, bio-ADM and sST2 significantly improve the $c$-statistic of the clinical model, reclassification analyses showed that all cardiovascular biomarkers, including hsTnI, improve predictive power of the multivariable model (Table 2).

\section{Discussion}

The FROG-ICU study confirmed the substantial number of vulnerable patients among ICU survivors. More importantly, FROG-ICU identified clinical and biological factors at the time of ICU discharge that were associated with an increased risk of long-term death.

We found that the 1-year mortality rate in ICU survivors was roughly $20 \%$, a figure comparable to that already described [8, 26-29]. The FROG-ICU study confirmed that increasing age and number of comorbidities are independently associated with an increased long-term risk of death [30]. In contrast to previous findings [31], with the exception of blood transfusion and prolonged length of ICU stay, we found no "in-ICU" factor was associated with an increased risk of post-ICU death. Indeed, the reason for ICU admission, illness severity scores at admission and/or use of invasive therapy, factors known to be associated with ICU mortality, were not associated with worse long-term outcomes in our 1570 consecutive ICU survivors, as described recently [32].

A major strength of the FROG-ICU study is the provision of a comprehensive clinical and biological evaluation of 


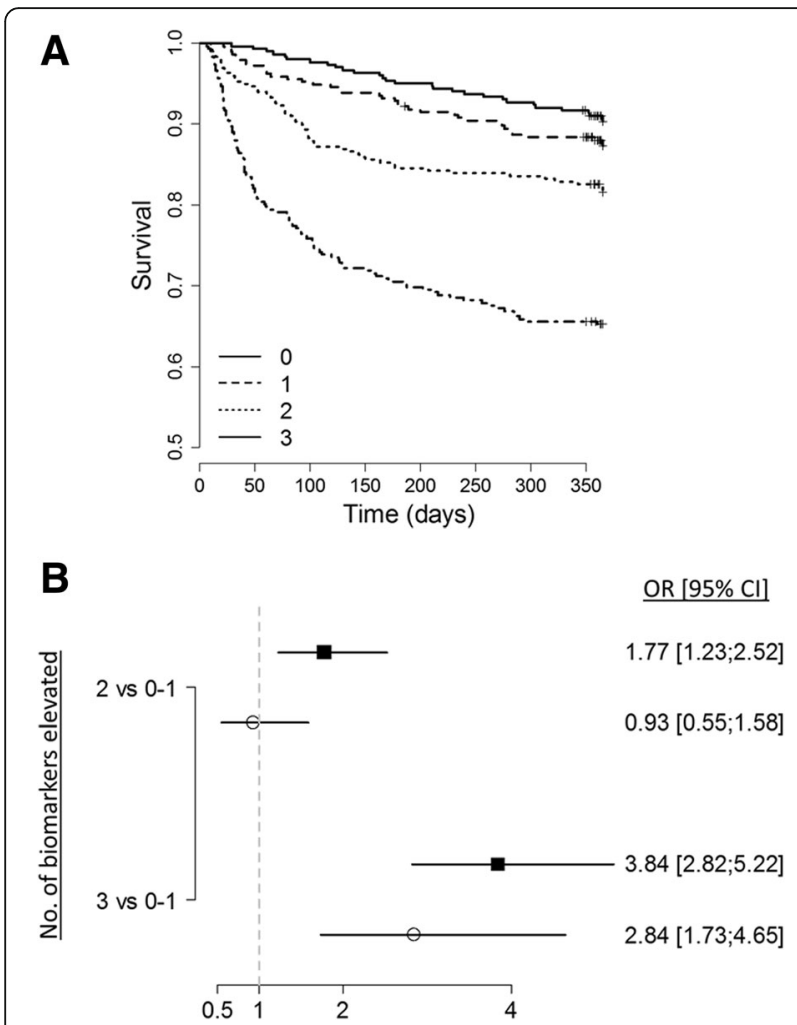

Fig. 3 Performance of cardiovascular biomarkers at ICU discharge to predict 1-year post-ICU survival. a Kaplan-Meier curves of patients discharged alive from the ICU according to the number of cardiovascular biomarkers elevated at discharge from the ICU. $\mathbf{b}$ Odds ratios for the risk of 1-year mortality according to the number of cardiovascular biomarkers elevated at discharge from the ICU. Biomarkers included NT-proBNP, sST2 and bio-ADM. Nonadjusted OR are presented as black squares and OR adjusted for the 14 variables of the multivariable model as white circles. OR odds ratio, $\mathrm{Cl}$ confidence interval

patients at the time of ICU discharge to assess risk prediction for subsequent poor outcomes. FROG-ICU demonstrated that hypotension and symptoms of persisting inflammation (abnormal temperature, protein, platelet and WBC count) were risk factors for a poor post-ICU outcome. FROG-ICU further showed that elevated biomarkers of impaired cardiac (NT-proBNP and sST2) and vascular (bio-ADM) function strikingly improved the prediction of post-ICU risk of death. Altogether, these data demonstrate that evidence of cardiovascular and/or inflammation abnormalities on ICU discharge is associated with, and likely leads to, a poor post-ICU outcome. Specific causes of death need to be ascertained but may be related to accelerated atheroma and plaque formation in the heart, brain or other organs, or repeated bouts of infection related to immunosuppression resulting from persisting inflammation. Those results are consistent with other work suggesting that the level of residual inflammation at discharge for patients with sepsis is associated with subsequent mortality [33].

\section{Limitations of the study}

Sixty-five (4\%) patients discharged alive from the ICU were not assessed at 1 year. Although the number is small, this could have affected the accuracy of our results. We cannot assess the risk of readmission after ICU discharge as this information was not recorded prospectively. More broadly, we had no information on patient management (e.g., drug therapy, rehabilitation, psychologist support) after ICU discharge. This may also have contributed to patient vulnerability and needs to be further explored. In addition, while we described clinical and biological variables independently associated with 1-year mortality in ICU survivors, other important parameters need to be considered when discharging a patient from the ICU, such as the amount of nursing care. Some potential predictors of post-ICU outcome were not considered in the present study; in particular, only comorbidities were considered but no frailty score. Moreover, because of the French law, we were not allowed to include patients with no social security coverage, which may limit the external validity of our results. Biological collection was performed when the patient physically left the ICU and not at the time the patient was considered dischargeable from the ICU, which is more tightly linked to the physiologic status of the patient. However, our approach reflects the real-life management of ICU discharge. Although the study was multicentric and conducted in two European countries, only one center outside France included patients; this may limit the external validity of our results. Finally, despite the fact that a sample size calculation was performed, factors that were weakly associated with the 1year risk of death could not be identified due to insufficient study power. Of note, the main aim of the study was to identify an explanatory model. Thus, the objective of our variable selection procedure was to identify the factors most strongly associated with mortality at 1 year and not to establish a prognostic score that would have to be validated.

\section{Conclusions}

Our findings suggest recommending a comprehensive clinical examination and targeted biological testing, including biomarker measures in ICU survivors, to guide personalized discharge long-term planning. Future trials should assess whether actions targeting the pathophysiology underlying the abnormal cardiac or vascular biomarkers may translate into improved post-ICU outcomes. In summary, the FROG-ICU study confirmed the striking prevalence of death at 1 year after ICU discharge. The FROG-ICU study further identified clinical and biological factors that may guide personalized discharge planning. 


\section{Additional file}

Additional file 1: Figure S1. showing Kaplan-Meier curves for 1-year mortality after discharge from the ICU, Figure S2. showing plots of restricted cubic spline of continuous variables included in the multivariable model, Figure S3. showing plots of restricted cubic spline of continuous variables included in the multivariable model, Table S1. presenting details on comorbidities and chronic treatment, and Table S2. presenting ORs (with 95\% Cl) for variables significantly associated with 1-year mortality in univariate analysis and in multivariable analysis (DOCX $110 \mathrm{~kb}$ )

\section{Abbreviations}

AUC: Area under the curve; bio-ADM: Bio-adrenomedullin; Cl: Confidence interval; eGFR: Estimated glomerular filtration rate; FROG-ICU: French and European Outcome reGistry in Intensive Care Units; hs-Tnl: Hyper-sensitive troponin I; ICU: Intensive care unit; IDI: Integrative discrimination index; IQR: Interquartile range; NRI: Net reclassification index; NT-proBNP: N-terminal pro-B type natriuretic peptide; OR: Odds ratio; PICS: Post-intensive care syndrome; ROC: Receiver operating curve; SAPS: Simplified Acute Physiologic Score; SOFA: Sequential Organ Failure Assessment; sST2: Soluble ST2;

WBC: white blood cell

\section{Acknowledgements}

The authors are particularly grateful to Marie-Céline Fournier who coordinated organizational aspects of the study. They also thank the Centre de Recherche Clinique (CRC) of Lariboisière University Hospital for support. Investigators for the FROG-ICU study

\begin{tabular}{|c|c|}
\hline Center & Investigators \\
\hline Hopital Lariboisiere (Paris) & $\begin{array}{l}\text { N Deye, C Fauvaux, A Mebazaa, C } \\
\text { Damoisel, D Payen }\end{array}$ \\
\hline Hopital Saint Louis (Paris) & E Azoulay, AS Moreau, L Jacob, O Marie \\
\hline Hopital Bichat (Paris) & M Wolf, R Sonneville, R Bronchard \\
\hline Hopital Beaujon (Clichy) & I Rennuit, C Paugam \\
\hline Hopital Cochin (Paris) & JP Mira, A Cariou, A Tesnieres \\
\hline $\begin{array}{l}\text { Hopital Bicetre (Le Kremlin- } \\
\text { Bicetre) }\end{array}$ & $\begin{array}{l}\text { N Dufour, N Anguel, L Guerin, J } \\
\text { Duranteau, C Ract }\end{array}$ \\
\hline Chu De Marseille (Marseille) & M Leone, B Pastene \\
\hline $\begin{array}{l}\text { Hopital Raymond Poincare } \\
\text { (Garches) }\end{array}$ & T Sharshar, A Fayssoyl \\
\hline Hopital Saint-Antoine & J-L Baudel, B Guidet \\
\hline $\begin{array}{l}\text { Hopital De La } \\
\text { Pitie_-Salpetriere (Paris) }\end{array}$ & Q Lu, W Jie Gu, N Brechot, A Combes \\
\hline Chu St Eloi (Montpellier) & S Jaber, A Pradel, Y Coisel, M Conseil \\
\hline $\begin{array}{l}\text { Hopital Ambroise Pare } \\
\text { (Boulogne) }\end{array}$ & A Veillard Baron, L Bodson \\
\hline Chu Caremeau (Nimes) & Jy Lefrant, L Elotmani, A Ayral, S Lloret \\
\hline $\begin{array}{l}\text { Hopital Jean Minjoz } \\
\text { (Besançon) }\end{array}$ & S Pily-Flouri, Jb Pretalli \\
\hline Clinique Saint-Luc (Belgium) & $\begin{array}{l}\text { Pf Laterre, V Montiel, Mf Dujardin, C } \\
\text { Berghe }\end{array}$ \\
\hline
\end{tabular}

\section{Funding}

The FROG-ICU study was funded by the Programme Hospitalier de la Recherche Clinique (AON 10-216) and by a research grant from the Société Française d'Anesthésie-Réanimation.
Abbot, Sphingotec, Roche Diagnostics and Critical Diagnostics provided unrestricted free kits to Assistance Publique-Hôpitaux de Paris to conduct biomarker analyses.

\section{Availability of data and materials}

AM had full access to all data in the study and takes responsibility for the integrity of the data and the accuracy of the data analysis.

\section{Authors' contributions}

Study concept and design: EG, AM, MR-R, EV. Acquisition of data: AC, ND, AV-B, SJ, CD, QL, XM, IR, EA, MLé, HO, BG, DF, AT, RS, PM, SPF, J-YL, JD, P-FL, $N B, X R$. Analysis and interpretation of data: EG, AM, KC, MM, J-ML, MR-R. Drafting of the manuscript: EG, AM. Critical revision of the manuscript for important intellectual content: BC, J-ML, BG, AV-B, MLe, MS. Statistical analysis: EG, MR-R. Obtained funding: EG, AM, EV. Administrative, technical or material support: EG, AM, EV. Study supervision: EG, AM. All authors read and approved the final manuscript.

\section{Ethics approval and consent to participate}

The study was conducted in France and Belgium in accordance with Good Clinical Practice (Declaration of Helsinki 2002) and Ethical Committee approvals (Comité de Protection des Personnes - lle de France IV, IRB n 00003835 and Commission d'éthique biomédicale hospitalo-facultaire de I'hôpital de Louvain, IRB nB403201213352).

\section{Consent for publication}

Not applicable

\section{Competing interests}

EG received research grant from Sphingotec, and consultancy fees from Magnisense and Roche Diagnostics. AM received speaker's honoraria from Abbott, Novartis, Orion, Roche and Servier, and fees as a member of the advisory board and/or Steering Committee from Cardiorentis, Adrenomed, MyCartis, Neurotronik and Sphyngotec. The remaining authors declare that they have no competing interests.

\section{Publisher's Note}

Springer Nature remains neutral with regard to jurisdictional claims in published maps and institutional affiliations.

\footnotetext{
Author details

${ }^{1}$ Department of Anesthesiology, Critical Care and Burn Unit, Hôpitaux Universitaires Saint Louis_Lariboisière, Assistance Publique-Hôpitaux de Paris, Université Paris Diderot_-Paris 7, Sorbonne Paris Cité, UMR-S 942, INSERM, Paris, France. ${ }^{2}$ Medical Intensive Care Unit, Cochin University Hospital, Assistance Publique-Hôpitaux de Paris, Paris Descartes University, Paris Cardiovascular Research Center-INSERM U970 (PARCC), Paris Sudden Death Expertise Center, Paris, France. ${ }^{3}$ Medical Intensive Care Unit, Hôpitaux Universitaires Saint Louis_Lariboisière, Assistance Publique-Hôpitaux de Paris, Université Paris Diderot_Paris 7, Sorbonne Paris Cité, UMR-S 942, INSERM, Paris, France. ${ }^{4}$ Intensive Care Unit, University Hospital Ambroise Paré Assistance Publique-Hopitaux de Paris, 26930 Boulogne-Billancourt, France. ${ }^{5}$ Intensive Care Unit, Anaesthesia and Critical Care Department, Saint Eloi Teaching Hospital, Centre Hospitalier Universitaire Montpellier, Montpellier University, Montpellier, France. ${ }^{6}$ Multidisciplinary Intensive Care Unit, Department of Anesthesiology and Critical Care Medicine, La Pitié-Salpêtrière Hospital, Assistance Publique Hôpitaux de Paris, UPMC Paris 6, Paris, France. ${ }^{7}$ Medical Intensive Care Unit, Bicêtre Hospital, Paris-Sud University Hospitals, Inserm UMR_S999, Paris-Sud University, Le Kremlin-Bicêtre, France. ${ }^{8}$ Department of Anesthesiology and Critical Care, Beaujon Hospital, Assistance Publique Hôpitaux de Paris University, Clichy, France. ${ }^{9}$ Medical Intensive Care Unit, Hôpital Saint-Louis, ECSTRA Team, Biostatistics and Clinical Epidemiology, UMR 1153 (Center of Epidemiology and Biostatistics Sorbonne Paris Cité, CRESS), INSERM, Université Paris Diderot Sorbonne, Paris, France. ${ }^{10}$ Service d'anesthésie et de réanimation, Hôpital Nord, Assistance Publique-Hôpitaux de Marseille, Aix Marseille Université, Marseille, France. ${ }^{11}$ Service de Réanimation Médicale, Hôpital Saint-Antoine, Assistance Publique-Hôpitaux de Paris, Université Pierre et Marie Curie, Paris, France. ${ }^{12}$ General Intensive Care, Raymond Poincaré University Hosptal, Assistance Publique-Hopitaux de Paris, Garches, France. ${ }^{13}$ Department of Anesthesiology and Intensive Care, Cochin University Hospital, Assistance
} 
Publique-Hôpitaux de Paris, Paris Descartes University, Paris Cardiovascular Research Center-INSERM U970 (PARCC), Paris Sudden Death Expertise Center, Paris, France. ${ }^{14}$ Department of Intensive Care Medicine and Infectious Diseases, Univ Paris Diderot, Sorbonne Paris Cité, Assistance Publique-Hôpitaux de Paris, Hôpital Bichat-Claude, Paris, France. ${ }^{15}$ Department of Anesthesiology and Intensive Care, Bichat University Hospital, Assistance Publique-Hôpitaux de Paris, Université Paris Diderot-Paris 7, Sorbonne Paris Cité, Paris, France. ${ }^{16}$ Department of Anesthesiology and Intensive Care Medicine, University Hospital of Besancon, 25000 Besancon, France. ${ }^{17}$ Department of Anesthesiology, Emergency and Critical Care Medicine, Nimes University Hospital, 30029 Nîmes, France. ${ }^{18}$ Physiology Department, EA 2992, Faculté de Médecine de Nîmes, Université Montpellier 1, 30029 Nîmes, France. ${ }^{19}$ Département d'Anesthésie-Réanimation, Hôpital de Bicêtre, Université Paris-Sud, Hôpitaux Universitaires Paris-Sud, Assistance Publique-Hôpitaux de Paris, Le Kremlin Bicêtre, Paris, France. ${ }^{20}$ Medical-Surgical Intensive Care Unit, Cliniques Saint-Luc, Brussels, Belgium. ${ }^{21}$ Medical Intensive Care Unit, Hôpital Pitié-Salpêtrière, Assistance Publique—Hôpitaux de Paris, Sorbonne Pierre-Marie Curie University Paris, INSERM, UMRS_1166-ICAN, Institute of Cardiometabolism and Nutrition and CIC 1421—Paris Est, Paris, France. ${ }^{22}$ URC-Eco, Assistance Publique-Hôpitaux de Paris, Sorbonne Paris Cité, Université Paris Diderot, ECEVE, INSERM, Paris, France. ${ }^{23}$ Department of Anesthesiology and Critical Care Medicine, Hôpital Européen Georges Pompidou, APHP, Université Paris Descartes, Sorbonne Paris Cite, Paris, France. ${ }^{24}$ Service de Biochimie, Hôpitaux Universitaires Saint Louis—Lariboisière, Assistance Publique—Hôpitaux de Paris, Université Paris Diderot-Paris 7, Sorbonne Paris Cité, UMR-S 942, INSERM, Paris, France. ${ }^{25}$ Unité de Recherche Clinique, Hôpitaux Universitaires Saint Louis—Lariboisière, Assistance Publique—Hôpitaux de Paris, Université Paris Diderot-Paris 7, Sorbonne Paris Cité, UMR-S 942, INSERM, Paris, France. ${ }^{26}$ Bloomsbury Institute of Intensive Care Medicine, University College London, Cruciform Building, Gower St, London WC1E 6BT, UK. ${ }^{27}$ Service de Biostatistique et Information Médicale, Hôpitaux Universitaires Saint Louis—Lariboisière, Assistance Publique-Hôpitaux de Paris, Université Paris Diderot-Paris 7, Sorbonne Paris Cité, ECSTRA Team, INSERM, Paris, France. ${ }^{28}$ Department of Anesthesiology and Intensive Care, University Paris Diderot, INSERM UMR-S 942, Saint Louis-Lariboisière University Hospitals, 2 rue Ambroise Paré, 75010 Paris, France.

Received: 7 August 2017 Accepted: 8 December 2017

Published online: 18 January 2018

\section{References}

1. Winters BD, Eberlein M, Leung J, Needham DM, Pronovost PJ, Sevransky JE. Long-term mortality and quality of life in sepsis: a systematic review. Crit Care Med. 2010;38(5):1276-83.

2. Needham DM, Davidson J, Cohen H, Hopkins RO, Weinert C, Wunsch H, Zawistowski C, Bemis-Dougherty A, Berney SC, Bienvenu OJ, et al. Improving long-term outcomes after discharge from intensive care unit: report from a stakeholders' conference. Crit Care Med. 2012;40(2):502-9.

3. Desai SV, Law TJ, Needham DM. Long-term complications of critical care. Crit Care Med. 2011;39(2):371-9.

4. Wright JC, Plenderleith L, Ridley SA. Long-term survival following intensive care: subgroup analysis and comparison with the general population. Anaesthesia. 2003;58(7):637-42.

5. Cuthbertson BH, Rattray J, Campbell MK, Gager M, Roughton S, Smith A, Hull A, Breeman S, Norrie J, Jenkinson D, et al. The PRaCTICaL study of nurse led, intensive care follow-up programmes for improving long term outcomes from critical illness: a pragmatic randomised controlled trial. BMJ. 2009:339:b3723.

6. Williams TA, Dobb GJ, Finn JC, Knuiman MW, Geelhoed E, Lee KY, Webb SA. Determinants of long-term survival after intensive care. Crit Care Med. 2008; 36(5):1523-30

7. Flaatten $\mathrm{H}$, Kvale R. Survival and quality of life 12 years after ICU. A comparison with the general Norwegian population. Intensive Care Med. 2001;27(6):1005-11.

8. Niskanen M, Kari A, Halonen P. Five-year survival after intensive care-comparison of 12,180 patients with the general population. Finnish ICU Study Group. Crit Care Med. 1996;24(12):1962-7.

9. Hofhuis JG, Spronk PE, van Stel HF, Schrijvers GJ, Rommes JH, Bakker J. The impact of critical illness on perceived health-related quality of life during
ICU treatment, hospital stay, and after hospital discharge: a long-term follow-up study. Chest. 2008;133(2):377-85.

10. Hodgson CL, Udy AA, Bailey M, Barrett J, Bellomo R, Bucknall T, Gabbe BJ, Higgins AM, Iwashyna TJ, Hunt-Smith J, et al. The impact of disability in survivors of critical illness. Intensive Care Med. 2017;43(7): 992-1001.

11. Dinglas VD, Aronson Friedman L, Colantuoni E, Mendez-Tellez PA, Shanholtz CB, Ciesla ND, Pronovost PJ, Needham DM. Muscle weakness and 5-year survival in acute respiratory distress syndrome survivors. Crit Care Med. 2017:45(3):446-53.

12. Garland A, Olafson K, Ramsey CD, Yogendran M, Fransoo R. Distinct determinants of long-term and short-term survival in critical illness. Intensive Care Med. 2014;40(8):1097-105.

13. Yende S, Linde-Zwirble W, Mayr F, Weissfeld LA, Reis S, Angus DC. Risk of cardiovascular events in survivors of severe sepsis. Am J Respir Crit Care Med. 2014;189(9):1065-74.

14. Mebazaa A, Casadio MC, Azoulay E, Guidet B, Jaber S, Levy B, Payen D, Vicaut E, Resche-Rigon M, Gayat E. Post-ICU discharge and outcome: rationale and methods of the The French and euRopean Outcome reGistry in Intensive Care Units (FROG-ICU) observational study. BMC Anesthesiol. 2015:15:143.

15. Marino R, Struck J, Maisel AS, Magrini L, Bergmann A, Di Somma S. Plasma adrenomedullin is associated with short-term mortality and vasopressor requirement in patients admitted with sepsis. Crit Care. 2014;18(1):R34.

16. Yancy CW, Jessup M, Bozkurt B, Butler J, Casey Jr DE, Drazner MH, Fonarow GC, Geraci SA, Horwich T, Januzzi JL, et al. 2013 ACCF/AHA guideline for the management of heart failure: a report of the American College of Cardiology Foundation/American Heart Association Task Force on Practice Guidelines. J Am Coll Cardiol. 2013;62(16):e147-239.

17. Lassus J, Gayat E, Mueller C, Peacock WF, Spinar J, Harjola VP, van Kimmenade R, Pathak A, Mueller T, Disomma S, et al. Incremental value of biomarkers to clinical variables for mortality prediction in acutely decompensated heart failure: the Multinational Observational Cohort on Acute Heart Failure (MOCA) study. Int J Cardiol. 2013;168(3):2186-94.

18. Rey G, Jougla E, Fouillet A, Hemon D. Ecological association between a deprivation index and mortality in France over the period 1997-2001: variations with spatial scale, degree of urbanicity, age, gender and cause of death. BMC Public Health. 2009;9:33.

19. Harrell Jr FE, Lee KL, Mark DB. Multivariable prognostic models: issues in developing models, evaluating assumptions and adequacy, and measuring and reducing errors. Stat Med. 1996;15(4):361-87.

20. Rubin D. Multiple Imputation for Nonresponse in Surveys. New York: Wiley; 1987.

21. Hosmer D, Lemeshow S. Applied Logistic Regression. New York: Wiley; 2000.

22. Hlatky MA, Greenland P, Arnett DK, Ballantyne CM, Criqui MH, Elkind MS, Go $A S$, Harrell Jr FE, Hong Y, Howard BV, et al. Criteria for evaluation of novel markers of cardiovascular risk: a scientific statement from the American Heart Association. Circulation. 2009;119(17):2408-16.

23. Pencina MJ, D'Agostino Sr RB, D'Agostino Jr RB, Vasan RS. Evaluating the added predictive ability of a new marker: from area under the ROC curve to reclassification and beyond. Stat Med. 2008;27(2):157-72.

24. Cook NR. Use and misuse of the receiver operating characteristic curve in risk prediction. Circulation. 2007;115(7):928-35.

25. le Cessie S, van Houwelingen HC. Testing the fit of a regression model via score tests in random effects models. Biometrics. 1995;51(2):600-14.

26. Rockwood K, Noseworthy TW, Gibney RT, Konopad E, Shustack A, Stollery D, Johnston R, Grace M. One-year outcome of elderly and young patients admitted to intensive care units. Crit Care Med. 1993;21(5):687-91.

27. Bagshaw SM, Mortis G, Doig CJ, Godinez-Luna T, Fick GH, Laupland KB. Oneyear mortality in critically ill patients by severity of kidney dysfunction: a population-based assessment. Am J Kidney Dis. 2006;48(3):402-9.

28. Orwelius L, Nordlund A, Nordlund P, Simonsson E, Backman C, Samuelsson A, Sjoberg F. Pre-existing disease: the most important factor for health related quality of life long-term after critical illness: a prospective, longitudinal, multicentre trial. Crit Care. 2010;14(2):R67.

29. Braun A, Chang D, Mahadevappa K, Gibbons FK, Liu Y, Giovannucci E, Christopher KB. Association of low serum 25-hydroxyvitamin D levels and mortality in the critically ill. Crit Care Med. 2011;39(4):671-7.

30. Hill AD, Fowler RA, Pinto R, Herridge MS, Cuthbertson BH, Scales DC. Longterm outcomes and healthcare utilization following critical illness-a population-based study. Crit Care. 2016;20:76. 
31. Wunsch H, Guerra C, Barnato AE, Angus DC, Li G, Linde-Zwirble WT. Threeyear outcomes for Medicare beneficiaries who survive intensive care. JAMA. 2010;303(9):849-56.

32. Herridge MS, Chu LM, Matte A, Tomlinson G, Chan L, Thomas C, Friedrich JO, Mehta S, Lamontagne F, Levasseur M, et al. The RECOVER Program: disability risk groups and 1-year outcome after 7 or more days of mechanical ventilation. Am J Respir Crit Care Med. 2016;194(7):831-44.

33. Yende S, D'Angelo G, Kellum JA, Weissfeld L, Fine J, Welch RD, Kong L, Carter M, Angus DC, Gen IMSI. Inflammatory markers at hospital discharge predict subsequent mortality after pneumonia and sepsis. Am J Respir Crit Care Med. 2008;177(11):1242-7.

Submit your next manuscript to BioMed Central and we will help you at every step:

- We accept pre-submission inquiries

- Our selector tool helps you to find the most relevant journal

- We provide round the clock customer support

- Convenient online submission

- Thorough peer review

- Inclusion in PubMed and all major indexing services

- Maximum visibility for your research

Submit your manuscript at www.biomedcentral.com/submit
Biomed Central 